\title{
A Descriptive Study to Assess the Knowledge and Attitude on Reproductive Health Among High School Girls in a Selected High School of Guwahati, Assam
}

\section{Kalpana Nath*}

Lecturer, Psychiatric Nursing, Regional College of Nursing, Guwahati, India

*Corresponding Author: Kalpana Nath, Lecturer, Psychiatric Nursing, Regional College of Nursing, Guwahati, India.
Received: October 05, 2020

Published: January 22, 2021

(C) All rights are reserved by Kalpana Nath.

\begin{abstract}
Introduction: Adolescents, often termed the "generation of hope", play a vital role in the health status of any country. Evidence from both developed and developing countries shows the importance of sexual reproductive education programs in preparing them for their transition to adulthood.

Methodology: A descriptive survey type of study was undertaken to assess the knowledge and attitude on reproductive health among 45 numbers of high school girls in Anand Academy located at Dispur, Assam using a structured questionnaire including sociodemographic characteristics and 30 items for assessing knowledge and a 10 items likert scale to assess attitude towards reproductive health.

Main findings: Among the 45 subjects, $86.66 \%$ girls have moderate level of knowledge and rest $13.44 \%$ have low level of knowledge, $60 \%$ have favourable and $40 \%$ have neutral attitude towards reproductive health. Positive association was found between sociodemographic variables and knowledge and attitude. Knowledge and attitude scores were found to be positively correlated.

Conclusion: Present study is limited only to the assessment of knowledge and attitude among only 45 high school girls of one school. Further, studies can be taken up with larger sample.
\end{abstract}

Keywords: Reproductive Health; Adolescent Girls; Knowledge; Attitude

\section{Introduction}

World Health Organization has defined adolescence as progression from appearance of secondary sexual characters to sexual maturity and development of adult mental processes. Puberty is the transitional period linking childhood to adulthood and involves physical, biological and psychosexual changes [1]. Adolescence includes age group between 10 to 19 years [2]. It is a period of rapid physical and biological changes which may lead to confusion, tension, frustration and feeling of insecurity. Adolescence is a prime time for health promotion and for establishment of healthy behaviour that will influence health in later years. This transition phase makes them vulnerable to a number of problems for example, psychosocial problems, general and reproductive health problems and sexually related problems (WHO).

The National population policy 2000 indentifies adolescents as an underserved group for which health, specifically reproductive and sexual health interventions are to be designed. The National Curriculum Framework 2005 explicitly highlights the need for integrating age appropriate adolescents reproductive and sexual health, including HIV/AIDS messages into the school curriculum. Adolescent reproductive health has been identified as a key strategy in the reproductive and Child Health (RCH-II) programme (RCH 2).

Reproductive health covers all aspects of adolescent health. It is an umbrella concept, consisting of several distinct, yet related issues such as abortion, child birth, sexuality, contraception and maternal mortality [3]. Biological, social, cultural, economical and behavioral factors play an important role in determination of reproductive health.

\section{Need of the study}

Adolescents comprise $20 \%$ of the world's total population. Out of 1.2 billion adolescents world-wide, about $85 \%$ live in developing 
countries. In India, there are 190 million adolescents comprising $21 \%$ of India's total population. Adolescent pregnancies constitute $10-15 \%$ of total pregnancies in India [4]. This is largely attributed to early marriage, a culture widely prevalent in the whole of Indian subcontinent, besides Africa [5]. The age at marriage is quite low in rural areas and adolescent girls in these communities fall into fertility trap quite early. These situations predispose girls to teenage pregnancy that may have more immediate effect on their life than any other problem. The changing moral and social values and shift in the standard of societal behavior from conservatism to liberal interaction between both sexes is attributed largely to exposure to the media especially television and movies. Adolescents find themselves sandwiched between a glamorous western influence and a stern conservatism at home, which strictly forbids discussion on sex. This dichotomy aggravates the confusion among adolescents. Among adolescents, girls are particularly more vulnerable [6].

In order to lead a healthy, responsible and fulfilling lives and protect themselves from reproductive health problems adolescents need to be knowledgeable about them and need adequate information about the physical, psycho-logical changes that take place during puberty, menstruation, pregnancy and child birth [7]. The need to address these problems through reproductive health education has been recognized at various national and international forums [8]. Among the several options available, creating awareness among adolescents appears to be an important tool. However adolescent education programmes in India face many challenges. A lot of debate is going on about how much of the reproductive health contents should be taught? Whether teachers should impart this knowledge or should it be imparted by health professionals [9].

Among adolescents, girls are biologically more susceptible to sexually transmitted diseases, including HIV/AIDS infection. A vast majority of adolescent girls in India are suffering from reproductive health morbidities [10]. Thus, in order to lead a healthy, responsible, fulfilling life and to have protection from reproductive health problems, adolescents need to have sound information about the physical, psychological and social changes that takes place though childhood and adolescence.

Thus, it is important to have information regarding level of knowledge related to reproductive health among adolescent girls, so that appropriate interventions can be planned.

Keeping the above facts in mind, the present study was planned which was aimed at assessment of knowledge and attitude on reproductive health so that need based educational intervention can be planned based on their lacking areas.

\section{Objectives}

- To assess the knowledge on reproductive health among high school girls in a selected high school of Guwahati, Assam

- $\quad$ To assess the attitude towards reproductive health among high school girls in a selected high school of Guwahati, Assam

- To find out the relationship between the knowledge of high school girls on reproductive health and their selected sociodemographic variables

- To find out the relationship between the attitude of high school girls towards reproductive health and their selected sociodemographic variable.

Methodology

- $\quad$ Research Approach: In view of the nature of the problem and to accomplish the intended objectives of the study, survey approach was considered most appropriate.

- Research Design: To fulfill the objectives of the study, a cross-sectional descriptive design was used for collection and analysis of data.

- Study Setting: The present study has been undertaken in the Anand Academy, a high school located at Dispur area of Guwahati, Assam.

- Study Population: The study population comprised of high school girls between 13 to 16 years of age who were studying in class VIII, IX and X in the Anand Academy, Guwahati, Assam.

- The Sample: The study sample comprised of 45 high school girls, 15 numbers from each classes VIII, IX and X respectively.

- Sampling Technique: Stratified random sampling technique was used to collect the required number of participants to form the sample for the present study. All the high school girls were divided into three strata based on their class of study i.e. class VIII, IX and X. Then 15 numbers of participants from each class was selected by lottery method.

\section{Inclusion criteria}

- High school girls who were studying in class VIII, IX, and X and were in the age group of 13 to 16 years.

Study variables: The study variables were: 
- Sociodemographic variables of high school girls like age, religion, education of father, education of mother, occupation of father, occupation of mother, number of siblings, type of family, age of attaining menarche and source of information regarding reproductive health.

Research variables were:

- Knowledge of high school girls on reproductive health

- Attitude of high school girls towards reproductive health.

\section{Definition of Terms}

- High school girls: Students in the age group of 13 to 16 years who were studying in class VIII, IX and X in the selected high school, Anand Academy.

- Reproductive health: Health related events regarding menstruation, fertilization, child birth, lactation, and responsible sexual behaviors.

- Knowledge: High school girl's score in the knowledge questionnaire on reproductive health.

- Attitude: High school girl's score in the attitude scale towards reproductive health.

Data Collection: Instruments and techniques

Literature was reviewed for the purpose of finding of appropriate tools to collect data for this study. Based on the objectives, the following data collection instruments and techniques were used to collect relevant data -

The tool is divided into 2 parts -

- Part I - Sociodemographic data sheet including age, religion, education of father, education of mother, occupation of father, occupation of mother, number of siblings, type of family, age of attaining menarche and source of information regarding reproductive health

- $\quad$ Part II - Questionnaire on assessment of knowledge regarding reproduction which consists of thirty (30) items.

- $\quad$ Part III- Likert type of attitude scale consisting of 10 items with maximum possible score 30 .

Data collection

- Formal permissions were obtained from the head of the institution i.e. the principal of the school.
- Informed consent was taken from the participants.

- Sociodemographic data sheet, Questionnaire on assessment of knowledge regarding reproductive health and attitude scale was distributed among the participants.

Data analysis and interpretation

Data were first tabulated and coded in a master data sheet. Frequency and percentage was computed for sociodemographic data as well as on items of knowledge questionnaire. Regression analysis was done to find out the relationship between the knowledge and attitude score on reproductive health and high school girl's sociodemographic variables.

\section{Results and interpretation}

Section I - Frequency and percentage distribution of sociodemographic data of participants.

The data presented in the above table indicated that majority of the participants were found to be of 14 years of age. Most of the participants belonged to Hindu religion. Majority of the participant's mother's education was found to be high school (35.6\%), graduate (33.3) and primary level (15.6\%) and maximum numbers of mothers were found to be housewives (64.4\%). In case of fathers of participants, maximum were found to have graduate level (68.9\%) education and have service (62.2\%). Majority of the study participants live in nuclear family (88.9). The age of attaining menarche was found to be 12 years in $40 \%, 11$ years in $22.2 \%, 13$ years in 20\%, 10 years in 11.1\%, 14 years in $4.4 \%$ and 9 years in only $2.2 \%$ of the study participants. Source of information regarding reproductive health was found to be a teacher in majority of the study participants (46.7\%), followed by mother in $28.9 \%$, doctor in $15.6 \%$ and books in $8.9 \%$ of the study participants.

Section II - Level of knowledge among high school girls on reproductive health

Data presented in the above table indicated that majority of the study participants have medium level (86.7\%) and only $13.3 \%$ have low level of knowledge regarding reproductive health. None of the participants have high level of knowledge on reproductive health. Score below $7\left(<25^{\text {th }}\right.$ percentile $)$ was considered low level, score above $22\left(>75^{\text {th }}\right.$ percentile) was considered as high and score between 10 and 20 (between $25^{\text {th }}$ and $75^{\text {th }}$ percentile) was considered moderate level of knowledge in the present study. 
A Descriptive Study to Assess the Knowledge and Attitude on Reproductive Health Among High School Girls in a Selected High School of Guwahati, Assam

\begin{tabular}{|c|c|c|c|}
\hline \multicolumn{2}{|c|}{ Parameter } & \multirow{2}{*}{$\begin{array}{c}\text { Frequency } \\
5\end{array}$} & \multirow{2}{*}{$\begin{array}{c}\text { Percentage \% } \\
11.1\end{array}$} \\
\hline Age & $13 y r s$ & & \\
\hline & $14 y r s$ & 22 & 48.9 \\
\hline & $15 y r s$ & 18 & 40 \\
\hline & $16 y r s$ & 0 & 0 \\
\hline \multirow[t]{3}{*}{ Religion } & Hindu & 38 & 84.4 \\
\hline & Islam & 7 & 15.6 \\
\hline & Christian & 0 & 0 \\
\hline \multirow[t]{6}{*}{ Education of mother } & Primary & 7 & 15.6 \\
\hline & Middle & 4 & 8.9 \\
\hline & High school & 16 & 35.6 \\
\hline & HS & 3 & 6.7 \\
\hline & Graduate & 15 & 33.3 \\
\hline & Post graduate & 0 & 0 \\
\hline \multirow[t]{4}{*}{ Occupation of mother } & Housewife & 29 & 64.4 \\
\hline & Business & 0 & 0 \\
\hline & Service & 7 & 15.6 \\
\hline & Professional & 9 & 20 \\
\hline \multirow[t]{6}{*}{ Education of father } & Primary & 1 & 2.2 \\
\hline & Middle & 0 & 0 \\
\hline & High school & 3 & 6.7 \\
\hline & HS & 9 & 20 \\
\hline & Graduate & 31 & 68.9 \\
\hline & Post graduate & 1 & 2.2 \\
\hline \multirow[t]{4}{*}{ Occupation of father } & Home maker & 0 & 0 \\
\hline & Business & 2 & 4.4 \\
\hline & Service & 28 & 62.2 \\
\hline & Professional & 15 & 33.3 \\
\hline \multirow[t]{3}{*}{ Type of family } & Nuclear & 40 & 88.9 \\
\hline & Joint & 5 & 11.1 \\
\hline & Extended & 0 & 0 \\
\hline \multirow[t]{5}{*}{ No. of sibling } & 0 & 1 & 2.2 \\
\hline & 1 & 20 & 44.4 \\
\hline & 2 & 18 & 40 \\
\hline & 3 & 2 & 4.4 \\
\hline & 4 & 4 & 8.9 \\
\hline \multirow[t]{6}{*}{ Age of attaining menarche } & 9 & 1 & 2.2 \\
\hline & 10 & 5 & 11.1 \\
\hline & 11 & 10 & 22.2 \\
\hline & 12 & 18 & 40 \\
\hline & 13 & 9 & 20 \\
\hline & 14 & 2 & 4.4 \\
\hline \multirow[t]{4}{*}{ Source of information } & Books & 4 & 8.9 \\
\hline & Doctor & 7 & 15.6 \\
\hline & Mother & 13 & 28.9 \\
\hline & Teacher & 21 & 46.7 \\
\hline
\end{tabular}

Table 1: Frequency and percentage distribution of sample characteristics $(n=45)$. 
A Descriptive Study to Assess the Knowledge and Attitude on Reproductive Health Among High School Girls in a Selected High School of Guwahati, Assam

\begin{tabular}{|c|c|c|}
\hline Level of knowledge & Frequency & Percentage \\
\hline Low $(<7)$ & 6 & $13.3 \%$ \\
\hline Medium( 7-22) & 39 & $86.7 \%$ \\
\hline $\operatorname{High}(>22)$ & 0 & $0 \%$ \\
\hline
\end{tabular}

Table 2: Frequency and percentage distribution of study participants in different level of knowledge regarding reproductive

$$
\text { health }(n=45) \text {. }
$$

Section II - Assessment of Level of attitude of high school girls on reproductive health

\begin{tabular}{|c|c|c|}
\hline Level of attitude & Frequency & Percentage \\
\hline Unfavorable $(<7)$ & 0 & $0 \%$ \\
\hline Neutral $(7-22)$ & 18 & $40 \%$ \\
\hline Favorable $(>22)$ & 27 & $60 \%$ \\
\hline
\end{tabular}

Table 3: Frequency and percentage distribution of study participants in different levels of attitude towards reproductive health $(n=45)$.
The above table depicted that majority of the study participants $(60 \%)$ have favorable and $(40 \%)$ have neutral attitude and no participants have unfavorable attitude towards reproductive health. Score below $7\left(<25^{\text {th }}\right.$ percentile $)$ was considered low unfavorable, score above $22\left(>75^{\text {th }}\right.$ percentile $)$ was considered as favorable and score between 10 and 20 (between $5^{\text {th }}$ to $75^{\text {th }}$ percentile) was considered neutral level of attitude in the present study.

Section IV - Relationship between the knowledge of high school girls on reproductive health and selected variables.

The above table indicates that there is a positive correlation between the knowledge regarding reproductive health and variables like age, education of father, education of mother, number of siblings, and age of attaining menarche. In contrast, negative relationship was found between knowledge and religion, occupation of father, occupation of mother, type of family, source of information on reproductive health and their attitude towards reproductive health. Strong positive relationship was observed between knowledge and age of study participants but no significant relationship

\begin{tabular}{|c|c|c|c|}
\hline Variables (X) & Values of co - relation & \multicolumn{2}{|c|}{ Knowledge Regression equations: Knowledge = A + BX } \\
\hline & & 12.935 & +1.032 \\
\hline Age & 0.731 & 1.180 & -0.018 \\
\hline Religion & 0.024 & 3.975 & +0.477 \\
\hline Education of father & 0.267 & 2.608 & +0.553 \\
\hline Education of mother & 0.181 & 2.862 & -0.217 \\
\hline Occupation of father & 0.174 & 1.661 & -0.081 \\
\hline Occupation of mother & 0.046 & 1.226 & +0.387 \\
\hline Siblings & 0.193 & 1.187 & -0.058 \\
\hline Type of family & 0.085 & 11.355 & +0.323 \\
\hline Age of attaining menarche & 0.137 & 3.251 & -0.090 \\
\hline Source of information & 0.042 & 1.65 & -0.041 \\
\hline Attitude & 0.039 & & \\
\hline
\end{tabular}

Table 4: Relationship between the knowledge on reproductive health among the study participants and various factors like age, religion, education of father, education of mother, occupation of father, occupation of mother, number of siblings, type of family, age of attaining menarche and source of information regarding reproductive health (correlation and linear regression analysis).

was observed with any factor which may be attributed to small sample size.

Section V - Relationship between the attitude of high school girls towards reproductive health and selected variables.

The above table indicates that there was a positive correlation between the attitude towards reproductive health and different variables like age, education of mother, occupation of father, number of siblings, and age of attaining menarche. In contrast, negative association was found between the attitude and different factors like religion, education of father, occupation of mother, type of family, source of information on reproductive health and knowledge on reproductive health. No significant relationship was observed which may be attributed to small sample size [11-16]. 
A Descriptive Study to Assess the Knowledge and Attitude on Reproductive Health Among High School Girls in a Selected High School of Guwahati, Assam

\begin{tabular}{|c|c|c|c|}
\hline \multirow{2}{*}{ Variables (X) } & \multirow{2}{*}{ Values of co - relation } & \multicolumn{2}{|c|}{ Attitude } \\
\cline { 3 - 4 } & & Regression equations: Attitude = A + BX \\
\hline Age & 0.014 & 1.452 & B \\
\hline Religion & 0.025 & 1.639 & -0.034 \\
\hline Education of father & 0.395 & 2.675 & -0.234 \\
\hline Education of mother & 0.113 & 1.493 & +0.069 \\
\hline Occupation of father & 0.189 & 1.187 & +0.160 \\
\hline Occupation of mother & 0.395 & 2.675 & -0.234 \\
\hline Siblings & 0.107 & 1.502 & +0.057 \\
\hline Type of family & 0.144 & 1.850 & -0.225 \\
\hline Age of attaining menarche & 0.166 & 0.724 & +0.074 \\
\hline Source of information & 0.028 & 1.644 & -0.014 \\
\hline Knowledge & 0.039 & 1.370 & -0.037 \\
\hline
\end{tabular}

Table 5: Relationship between the attitude towards reproductive health among the study participants and various factors like age, religion, education of father, education of mother, occupation of father, occupation of mother, number of siblings, type of family, age of attaining menarche and source of information regarding reproductive health (correlation and linear regression analysis).

\section{Discussion}

The present study aimed to assess the level of knowledge and attitude on reproductive health among the high school girls. It showed that majority of the participants (48.9) were 14 years of age. Different age groups were observed in different study for assessment of knowledge on reproductive health, such as, Padhy G.K., et al. (2013) found that majority of girls $(60.4 \%)$ were 15 years of age; Naidu S.A., et al. (2014) found mean age of girls 13.6+/). 86 years. Most of the participants belonged to Hindu religion. This finding corroborated with Padhy G.K., et al. (2013) where most of the girls (94.4\%) were Hindu.

Majority of the participant's mother's education was found to be high school (35.6\%), graduate (33.3) and primary level (15.6\%) and maximum mothers were found to be housewife (64.4\%). In case of fathers of study participants, maximum were found to have graduate level (68.9\%) education and have service (62.2\%). Majority of the study participants belonged to nuclear family (88.9\%). The age of attaining menarche was found to be 12 years in $40 \%$, 11 years in $22.2 \%, 13$ years in $20 \%, 10$ years in $11.1 \%, 14$ years in $4.4 \%$ and 9 years in only $2.2 \%$ of the study participants. Naidu S.A., et al. (2014) results reflected the mean age of attaining menarche to be 11.5+/-3.7; Dube shubha., et al. (2012) found majority of the participants attained menarche at 13 years in case of urban and 12 years in case of rural girls.
Source of information regarding reproductive health was found to be teacher in majority of the study participants (46.7\%), followed by mother in $28.9 \%$, doctor in $15.6 \%$ and books in $8.9 \%$ of the study participants. Dube shubha., et al. (2012) found that source of information in majority (52\%) was family.

The findings of the study indicated that majority of the study participants have medium level (86.7\%) only $13.3 \%$ have low level of knowledge regarding reproductive health and no participants have high level of knowledge on reproductive health. Only $25 \%$ of the girls answered correctly about the organ responsible for menstruation, and half of the participants responded that uterus is site of fetal growth. These findings corroborated with the findings of Naidu S.A., et al. (2014).

The findings of the study indicated that majority of the study participants (60\%) have favorable and (40\%) neutral attitude and no participants have unfavorable towards reproductive health.

The findings of the study indicated that there was a positive correlation between the knowledge on regarding reproductive health and variables like age, education of father, education of mother, number of siblings, and age of attaining menarche. In contrast to it, a negative relationship was found between knowledge and religion, occupation of father, occupation of mother, type of family, 
source of information on reproductive health and their attitude towards reproductive health. Strong positive relationship was observed between knowledge and age of study participants but no significant relationship was observed with any factor which may be attributed to small sample size.

The results of the study also indicated that there is a positive correlation between the attitude towards reproductive health and different variables like age, education of mother, occupation of father, number of siblings, and age of attaining menarche. In contrast to it negative association was found between the attitude and different factors like religion, education of father, occupation of mother, type of family, source of information on reproductive health and knowledge on reproductive health. No significant relationship was observed which may be attributed to small sample size.

\section{Conclusion}

It was observed that none of the participants have high level of knowledge on reproductive health, though they are studying in high school level wherein many of the components of reproductive health is incorporated in their syllabus. In societies like ours, parents and teachers hesitate to discuss reproductive health issues with their children. Great effort is needed in various ways to bridge the gap among adolescent girls regarding important reproductive health issues. Training programmes to school teachers by health professionals, involving parents in the health education programmes are some important steps that can be taken. Present study is limited only to the assessment of knowledge and attitude among only 45 high school girls of one school. Further, studies can be taken up with larger sample.

\section{Bibliography}

1. The second decade: improving adolescent health and development. World Health Organisation, Geneva: WHO (2001): 1-20.

2. INCLEN International Clinic Epidemiology Network. 19982001.

3. Sharma P., et al. "Problems related to menstruation among adolescents girls". Indian Journal of Pediatric 75.2 (2008): 125129.

4. UNICEF- India (the state of the world's children 2011).

5. Bhatia BD and Chandra K. "Adolescent mother and an un-prepared child". Indian Journal of Child Health 4 (1993): 67-70.

6. Benson MD., et al. "Sex education in the inner setting learning and retention". Journal of the American Medical Association 225 (1986): 43-47.
7. Parwej S., et al. "Reproductive health education intervention trial”. Indian Journal of Pediatric 72 (2005): 287-291.

8. Mandal K. "Teaching adolescent school girls about menstrual hygiene". Indian Journal of Nursing and Midwifery 1 (1998): 19-26.

9. Kothari P. "The need of sex education for adolescents in India". Report of national seminar, New Delhi: national council of education Research and training (1994).

10. Agarwal S., et al. "A study of knowledge and attitude of adolescent girls towards reproductive health and related problems". Indian Journal of Preventive and Social Medicine 38.1,2 (2007): 36-41.

11. "Adolescence: development and obstacles. Adolescence, research and reference centre, psychological aspects of AIDS CMC Vellore". Health Dialogue 28 (2002): 1-3.

12. "Adolescence, the critical phase, the challenges and the potential". World health organization (1997).

13. "Coming of age" from facts to action for adolescent sexual reproductive health. WHO/FRH/ADH/97.18, Geneva (1997): 4

14. Improving adolescent health and development. The second decade WHO/FRH/ADH/98.

15. Sharma V and Sharma A. "The letter box approach - A model for sex education in an orthodox society". Journal of Family Welfare 41 (1995): 31-33.

16. Adolescence, the critical phase, the challenges and the potential. World Health organization (1997).

\section{Assets from publication with us}

- Prompt Acknowledgement after receiving the article

- Thorough Double blinded peer review

- Rapid Publication

- Issue of Publication Certificate

- High visibility of your Published work

Website: $\underline{w w w . a c t a s c i e n t i f i c . c o m / ~}$

Submit Article: www.actascientific.com/submission.php Email us: editor@actascientific.com Contact us: +919182824667 